\title{
An Automated Robot-Car Control System with Hand- Gestures and Mobile Application Using Arduino
}

\author{
Saleem Ullah ${ }^{1}$, Zain Mumtaz ${ }^{1}$, Shuo Liu ${ }^{2}$, Mohammad Abubaqr ${ }^{1}$, Athar Mahboob ${ }^{3}$ and Hamza \\ Ahmad Madni2,3,* \\ 1 Department of Computer Science, Khwaja Fareed University of Engineering and Information Technology, \\ Rahim Yar Khan 64200, Pakistan; saleem.ullah@kfueit.edu.pk (S.U.); zainmumtaz007@gmail.com (Z.M.); \\ mohammadabubaqr@outlook.com (M.A) \\ 2 State Key Laboratory of Millimeter Waves, Department of Radio Engineering, Southeast University, \\ Nanjing 210096, China; liushuo.china@seu.edu.cn (S.L.); 101101770@seu.edu.cn (H.A.M.) \\ 3 Department of Computer Engineering, Khwaja Fareed University of Engineering and Information \\ Technology, Rahim Yar Khan 64200, Pakistan; athar@kfueit.edu.pk (A.M.) \\ * Correspondence: 101101770@seu.edu.cn; Tel.: +86-1782-6500-615 or +92-313-4820-856
}

\begin{abstract}
Gesture recognition has always been a technique to decrease the distance between the physical and the digital world. In this work, we introduce an Arduino based vehicle system which no longer require manual controlling of the cars. The proposed work is achieved by utilizing the Arduino microcontroller, accelerometer, RF sender/receiver, and Bluetooth. Two main contributions are presented in this work. Firstly, we show that the car can be controlled with hand-gestures according to the movement and position of the hand. Secondly, the proposed car system is further extended to be controlled by an android based mobile application having different modes (e.g., touch buttons mode, voice recognition mode). In addition, an automatic obstacle detection system is introduced to improve the safety measurements to avoid any hazards. The proposed systems are designed at lab-scale prototype to experimentally validate the efficiency, accuracy, and affordability of the systems. We remark that the proposed systems can be implemented under real conditions at large-scale in the future that will be useful in automobiles and robotics applications.
\end{abstract}

Keywords: Android; arduino; bluetooth; hand-gesture recognition; low cost; open source; sensors; smart cars; speech recognition

\section{Introduction}

A robot is an intelligent machine that is commanded by a computer application to perform various operations and services. Robots play an essential role in automation across all sectors like manufacturing, military, construction, and medical [1] etc. Robots not only helps humans to save time but also use to increases the productivity [2], efficiency, reliability, reduces the use of resources to save energy, and reduce the running cost etc. Meanwhile, robots play a significant role in providing help in such tasks that cannot be done smoothly by disabled persons, i.e., controlling the car by physical devices has become very successful. There are two categories of robots such as: autonomous robots (edge sensing robots [3], line sensing [4]) and remote-controlled robots (gesture-controlled robots [5]). Therefore, the employment of gesture-controlled robots is one of the most elegant and aesthetic term to catch the human-gesture that is difficult to understand by machine.

There are many methods to capture gesture commonly using data glove [6], camera [7], infrared waves [8], tactile [9], acoustic [10] and with motion technological means [11]. These embedded systems [6-11] are designed for particular control and can be optimized to increase reliability, performance and reduce the cost and size of the device. Moreover, researchers are showing tremendous interest in gesture recognition, building robots and many other devices that are directly controlled by human gestures. Gesture control mechanism is applied in various fields like socially assistive robots, augmented reality, emotion detection from facial expressions, recognition of sign languages [12-14] etc. Furthermore, the emotional gesture [12] identification from the face is also been investigated. Similarly, with the arrival of smartphone and other advanced technologies, 
operating machines have become more adaptable. In this regard, the smartphone communicates with the microcontroller of robot via radio frequency $(\mathrm{RF})$, Bluetooth or Wi-Fi.

The traditional robot system has been limited to the remote system in which the desired actions can only be done with the unique and specific remote. In this scenario, if the remote is broken or lost, the robot loses control and leads to hazards and waste of money as well. To overcome this remotecontrolling concept, controlling the robot using gesture recognition technique [15-33], Bluetooth android application [34-41], and voice recognition [42-50] have been proposed. Thus, in previous literature, most of them are line-sensing robots [51-53] that are controlled with Infrared LEDs, HF band short-range RFID systems [54] and CNY 70 sensor [55]. In addition, vision-based technology $[56,57]$ are also used to control the movement of the robot. Furthermore, the automated robot control system with six sense [58-60], and ZigBee based system [61-64] have also been implemented. In addition, another approach has been introduced to avoid robot from obstacles [65-67] in which the robot stops moving or changes its route of motion.

Apart from the traditional robot control system, the term "internet of things" (IoT) [68-74] is also essential for connecting robot with the internet to allow users to control robot from anywhere and anytime. These wireless systems are providing vital help to robot self-regulation systems by using $\mathrm{Wi}-\mathrm{Fi}$ and cloud computing, etc. As far as we know, a need still exists for the design of an automated robot control system that supports hand-gesture recognition, android mobile application control and voice recognition concepts, while monitoring the obstacles.

In this paper, we introduce the design and experimentally demonstrate a robot-car that can be controlled with hand movement by using the technique of hand-gesture. This work is accomplished with the conventional arrangements of the Arduino microcontroller, accelerometer, RF transmitter / receiver, Android mobile-application, Bluetooth and motor module. The proposed robot-car is controlled via gesture recognition technique by measuring the angles and position of the hand. In this way, the robot will move according to the movement of the hand. Furthermore, we extend this system to allow the robot to be controlled by just a click on the cellphone with an Android operating system, and the voice recognition via Bluetooth technology. There are two main controlling ways in android application. First one is the touch buttons, the robot will move accordingly as the user touches the button. The second one is voice recognition; the robot will follow and move accordingly as the user says the operating command. Most importantly, an obstacle detection sensor is set to detect the obstacle in front of it, and when sensor detects the obstacle, it stops moving. Hence, the proposed systems of controlling the robot car with both gesture control and android application control are performed and displayed at lab-scale prototype to confirm that the proposed designs can be easily implemented in the large and real-scale conditions in future.

The remaining content of the paper is ordered as follows. In Section 2, the idea of the automatic control robot car is introduced with a detailed explanation of the electronic components that are used in the proposed system, based on the gesture recognition and with the mobile-based android application. Also, the experimental results of proposed systems at lab-scale prototype are presented in Section 3, and Section 4 concludes the paper.

\section{Materials and Methods}

For the simplicity of analysis, Figure 1 demonstrates the complete working mechanism and the features of the proposed automatic robot car. Whereas, I/P and O/P represent the flow of the system as input and output. There are two modes of transmission and controlling of robot car. The first one is the hand-gesture system. The hand accelerometer first senses the acceleration forces from the direction of hand and send it to Arduino Nano that is attached with hand. After receiving the data, Arduino Nano converts it into different angle lies between $0^{\circ}-450^{\circ}$ and send it to the RF receiver of the Arduino Uno that is attached with the robot car through the RF sender. 


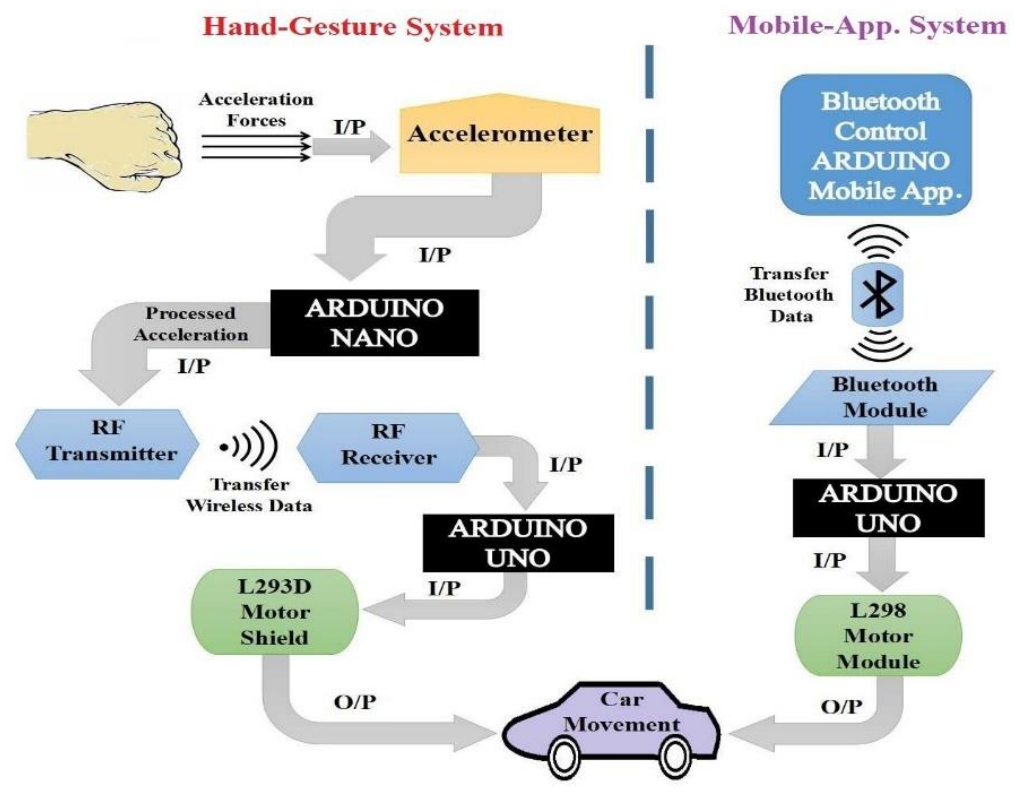

Figure 1. The architecture design of car controlling with hand-gesture and mobile application system.

After receiving the data, the Arduino Uno of car will measure the received angles with the predefined set of angles and send a signal to the motor module to move the wheels of the robot car accordingly to the angles. It can be noticed that the range of angles are predefined for the wheel movement of the robot car to move forward, backward, brake, left and right.

The second mode is to control a robot car with an android mobile application, which is built in android mobile application and available at google play store that can be easily downloaded [75]. In this system, when the user presses the corresponding touch button, a signal is transferred to the Arduino UNO that is attached to the car through the built-in mobile Bluetooth device. After receiving the following signal command, Arduino will check this signal with a predefined instruction that is programmed in coding and send the following signal to the motor module to move the wheels of robot car accordingly to the received signal.

\subsection{Electronic Components}

Various electronic components are used for creating electronic circuits. Consequently, our proposed circuit diagrams also contain those components that are specified in Table 1.

Table 1. Specification of electronic components used in to design the proposed system.

\begin{tabular}{cc}
\hline Components & Specifications \\
\hline Arduino UNO [50,76] & 28 pins; Operating voltage: 7-12V \\
Arduino Nano [77] & 30 pins; Operating voltage: 7-12V \\
MPU6050 Accelerometer [78] & 8 pins; Operating voltage 3.3V \\
RF Sender/Receiver [79] & Sender (3 pins; Operating voltage 3-12V; Transmission \\
& range: 90m), Receiver ( 4 pins, Operating voltage 5VDC) \\
L293D Motor Shield [80] & Supply-Voltage Range: 4.5-36V; Output current: 600 \\
mA/channel & Android compatible \\
Bluetooth Module HC-05 [81] & 6 pins; Operating voltage: 3.3-5V; Transmission range: 100m \\
Android Mobile Application [75] & Operating voltage: 5V; Max power: 25W \\
L298 Motor Module [82] &
\end{tabular}

\subsubsection{Arduino UNO}

The Arduino Uno [50,76] is a microcontroller board that is principally based on the ATmega328 microcontroller's series and has an integrated development environment (IDE) to write, compile, and uploads the programming codes to the microcontroller. Various sensors will forward the environmental data as an input to the microcontroller which will correspondingly send the attached 
peripherals, e.g., actuators. It has a total number of 28pins; 14 digital input/output pins (six are pulse width modulation (PWM) pins) and six pins are analogs used for interaction with the electronic components like sensors, motors; 3 GND pins (for grounding) and remaining pins for $3.3 \mathrm{~V}, 5 \mathrm{~V}$, VIN, RESET and AREF (Analogue Reference). Arduino contains a microcontroller with the 32KB storage memory, $2 \mathrm{~KB}$ of SRAM (Static random-access memory) and $1 \mathrm{~KB}$ of EEPROM (Electrically Erasable Programmable Read-Only Memory). Arduino primarily supports $C$ programming language compiler, macro-assemblers, and evaluation kits. It also has $16 \mathrm{MHz}$ ceramic resonators, a USB connection jack for connecting with computer, a jack for external power supply, an ICSP (in-circuit serial programmer) header, a reset button to reset to factory setting. It operates with an input voltage of 7 to $12 \mathrm{~V}$ (limit up to 20V).

\subsubsection{Arduino Nano}

Arduino [77] comprises of a microcontroller of ATmega328P with 32KB memory, of which 2KB is used for the bootloader, $2 \mathrm{~KB}$ of Static random-access memory (SRAM), 16MHZ Clock Speed, $1 \mathrm{~KB}$ of electrically erasable programmable read only memory (EEPROM). ATmega328P supports $C$ compiler, evaluation kits, and macro-assemblers. It has total 30 pins of which 14 are digital input and output pins (six are pulse width modulation (PWM)), 8 Analog IN pins, 2 GND pins, 2 RESET and remaining pins for VIN, 5V, $3 \mathrm{~V}$ and REF respectively. It has a USB connection jack, an external power supply jack, an ICSP header and a reset button. It operates with an input voltage of 7 to $12 \mathrm{~V}$ (limit up to $20 \mathrm{~V})$.

\subsubsection{MPU-6050 Accelerometer}

The MPU-6050 sensor [78] holds a MEMS (Micro-Electro-Mechanical Systems) accelerometer and a MEMS gyroscope only in the single chip. It contains 16-bits analogue to digital conversion hardware for each of the channels and it captures the $x, y$, and $z$ channel at the same time. MPU-6050 interfaces with the Arduino microcontroller using the I2C protocol. It has a total number of eight pins named as VCC ( for provides power), ground ( for grounding of system), serial clock (SCL used for providing clock pulse for I2C communication), serial data ( SDA used for transferring data through I2C communication), auxiliary serial data (XDA is optional but can be used to interface other I2C modules with MPU6050), auxiliary serial clock (XCL is also optional but can be used to interface other I2C modules with MPU6050), AD0 (If more than one MPU6050 is used a single MCU, then this pin can be used to vary the address). Interrupt pin (INT) indicates that data is available for MCU to read, and operating voltage is $3 \mathrm{~V}-5 \mathrm{~V}$.

\subsection{4. $\mathrm{RF}$ transmitter and receiver}

RF modules are $433 \mathrm{MHz}$ RF transmitter and receiver modules [79] that are used to transmit and receive the infrared waves. RF transmitter consists of three pins; ATAD (Signal pin used to send data for the receiver), VCC (for providing voltage) and Ground pin (for grounding the module). Its working voltage is $3 \mathrm{~V}-12 \mathrm{~V}$ and consumes $12 \mathrm{~V}$ power. RF transmitter can transmit up to $90 \mathrm{~m}$ in open area. RF Receiver module consists of four pins; VCC (for providing Voltage), two DATA pins for receiving data from transmitter and Ground (for grounding the module). Its working voltage is 5VDC.

\subsubsection{L293D}

The L293D [80] is quadruple high-current half- $\mathrm{H}$ drivers which are used to control the direction and speed of up to four direct current (DC) motors with individual 8-bit speed selection simultaneously of up to $0.6 \mathrm{~A}$ each. It is designed to provide bidirectional drive currents of up to 600$\mathrm{mA}$ at voltages from 4.5 to $36 \mathrm{~V}$. It has eight output pins for connecting four DC motors or two Stepper motors, one reset button, six pins for connecting two servo motors, one $+\mathrm{M}$ pin for providing external motor power, five Analog input pins, thirteen digital input pins for connecting with Arduino and ground pins for grounding.

\subsubsection{Bluetooth Module HC-05}

The HC-05 [81] is a Bluetooth SPP (Serial Port Protocol) module which is designed for direct wireless serial connection setup and can be used in a Slave or Master configuration, giving it an 
outstanding solution for wireless transmission. This serial port Bluetooth module is entirely adequate Bluetooth V2.0+EDR (Enhanced Data Rate) 3Mbps Modulation with complete 2.4GHz radio transceiver and baseband. It has a total number of six pins; ENABLE pin to toggle between Data Mode and AT command mode, VCC pin for providing voltage, Ground pin for grounding, TX-Transmitter for transmitting serial data, RX-Receiver for receiving the serial data and a State pin (to check if Bluetooth is working correctly or paired/unpaired). Its input voltage is $3.3-5 \mathrm{~V}$ and can transmit up to 90 meters.

\subsubsection{Android Mobile Application}

An Android application is a software application (developed with a computer programming language) which will run only on the Android platform because the Android platform is developed for mobile/tablet devices. A typical Android application is designed for a smartphone or tablets which operates on the Android OS. The application for controlling robot car is available at [75] and easily downloadable.

\subsubsection{L298N motor module}

An L298N motor module [82] is a heavy-duty dual H-bridge controller, which is used to control the direction and speed of single or two direct current (DC) motors of up to 2A each, having a voltage between $5 \mathrm{~V}$ to $35 \mathrm{~V}$. It has principally four output pins for the connection of the DC motors, four input pins to receive the signal from the microcontroller, two enable jumpers (remove one of the corresponding jumpers and connect to the pulse width modulation pins to control the speed of DC motors). It also has an onboard $5 \mathrm{~V}$ regulator, removes that regulator if the supply voltage is up to $12 \mathrm{~V}$.

\section{Designing Methodology}

\subsection{Hand-Gestures Recognition}

Figure 2 shows the circuit design of hand-gestures, which control the robotic car using hand movement. In this scenario, the robotic car will move in the same direction as the direction of hand rotation.

There are two schematic diagrams for the hand-gesture. Figure $2 \mathrm{a}$ is for the hand-gesture setup and Figure $2 b$ is for the car setup. First, we describe the hardware implementation of the hand-gesture setup. In this task, one Arduino Nano, one MPU-6050 accelerometer and one RF transmitter were used. The SLC pin of the MPU-6050 module is connected to the Arduino analogue Pin A5, SDA to the A4, Ground pin to the GND and VCC (voltage at the common collector) pin to the $5 \mathrm{~V}$ pin of the Arduino. DATA pin of the RX-transmitter is connected to the Arduino digital PIN D8, VCC pin to 5V and ground pin to GND port, as shown in Figure 2a.

After hardware implementing of the hand gesture, we move to the implementation of car setup. In this task, one L293D motor module, one Arduino Uno and one RF-receiver were used. Digital pins from A0-A13 of L293D motor module are connected to the digital pins A0-A13 and Analog pins A0A5 are connected to the analog pins A0-A5, and similarly, Vin pin to Vin port, 3V pin to $3 \mathrm{~V}$ port, $5 \mathrm{~V}$ pin to $5 \mathrm{~V}$ port, reset pin to reset port, ground pin to GND port and AREF pin to the AREF port of Arduino Uno as displayed in Figure 2b. Further, M1.1-M4.1 pins are connected to the negative terminal and M1.2-M4.2 pins of the motor module are connected to the positive terminals of the motor. Similarly, VCC pin of RF-receiver is connected to the VCC pin of the motor module, DATA pin to the digital pin D2 of motor module and Arduino and at last, the Ground pin to the GND port of Arduino Uno. The complete software code of this case is presented in Figure S1 of the supplementary materials. 
a) Hand-Gesture Setup

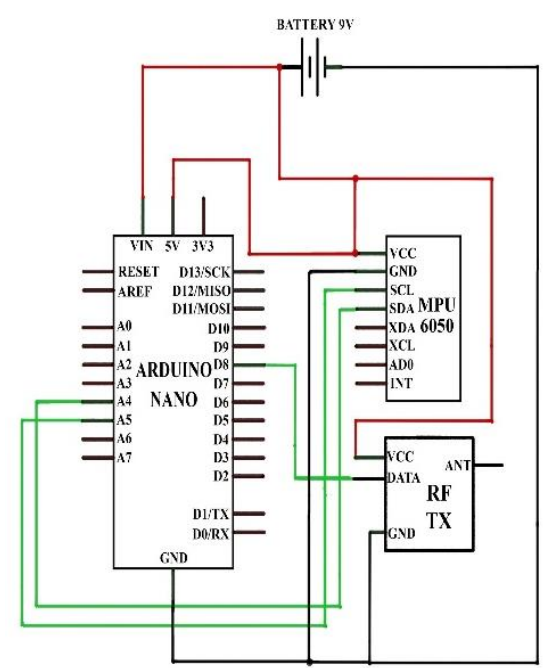

b) Car Setup

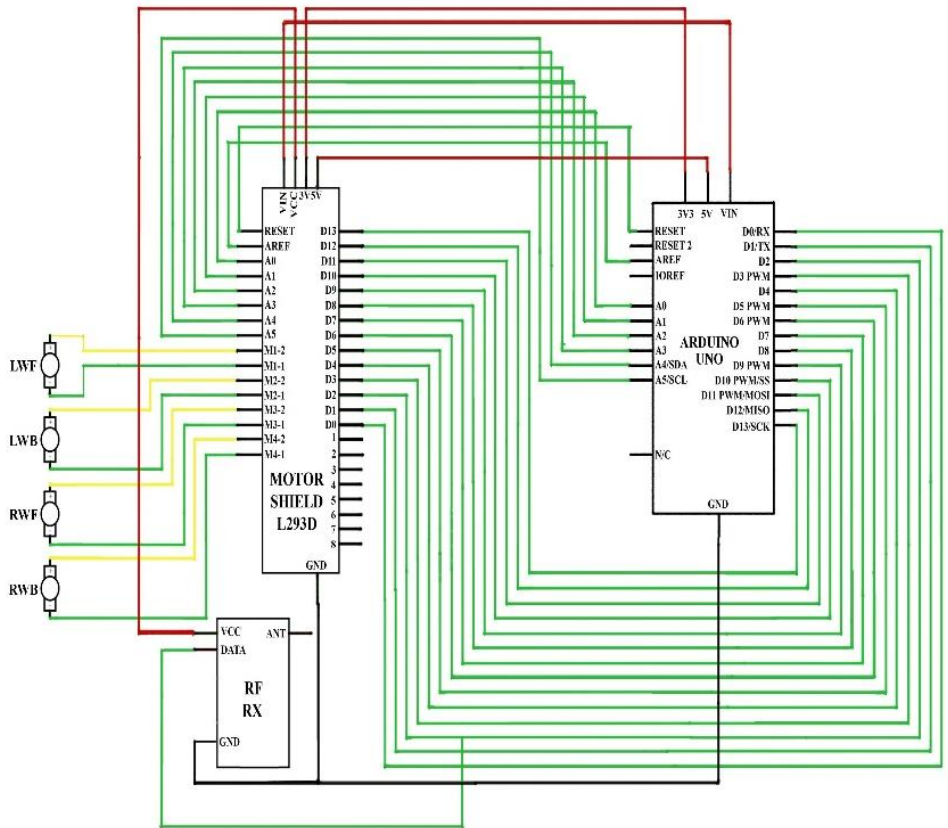

Figure 2. Circuit design of car controlling system using hand gestures recognition. (a) Shows the schematic of hand-gesture setup. (b) Display the schematic design of car setup.

\subsubsection{Movement of Motors with Hand-Gesture}

As the user moves own hand, the reading of the accelerometer will change, and then it will be recaptured by the application. There are genuinely two values: One is the minimum value $\left(\mathrm{X}_{\mathrm{range}}\right)$, and another is the maximum value ( $\left.Y_{\text {range }}\right)$, and the range is defined using these two values for each function of the car. In simple words, the set of ranges are defined for the movement of the robot car in a specific way. If the received data by the application lies within these specified values, then the corresponding decision will be made. This decision value will be sent to the microcontroller, which then processes it to understand the corresponding gesture. After knowing the gesture, it will send a signal to move the robot car accordingly. There is a total of four DC motors, one motor for each wheel (two for left diagonal wheels, and two for right diagonal wheels) used in the construction of this car. The motors are controlled by the L293D motor shield.

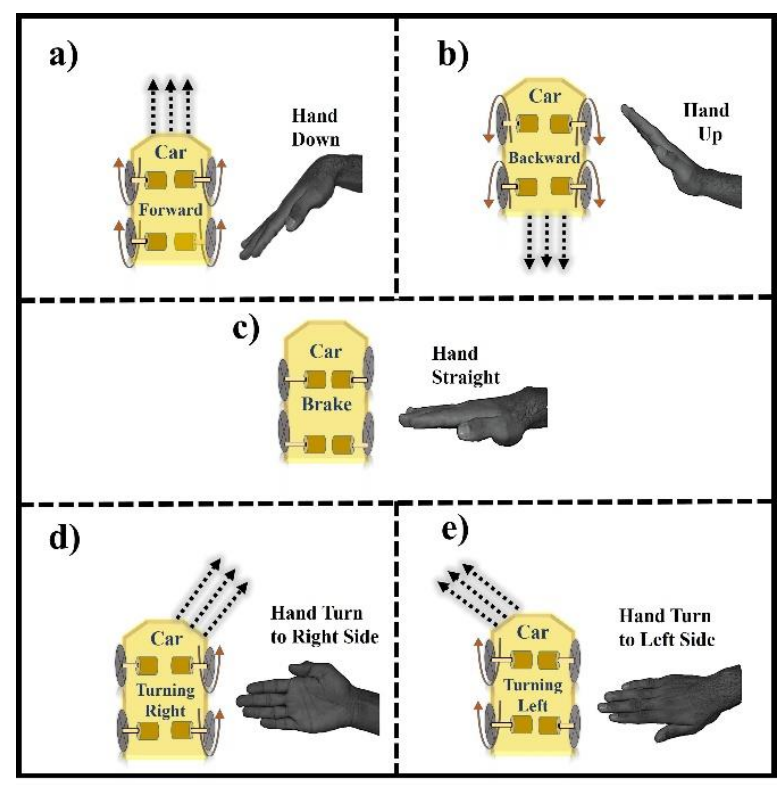

Figure 3. The theme of gesture recognition (i.e., movement of hand, motors and wheels).

Figure 3 represents the main idea of gesture recognition and car movement. When the user tilts his hand downward, the gesture is recognized as the forward movement, all the four wheels of motors 
will rotate forward and the robot will move in the forward direction which can be easily seen in the Figure $3 \mathrm{a}$. Figure $3 \mathrm{~b}$ illustrates the case when the user tilts his hand upwards, the gesture is recognized as the move backward gesture, and all the four wheels of motors will rotate backward, and the robot moves in the backward direction. In Figure $3 c$, the hand is in the straight position, and the gesture is recognized as to stop the car, all the four wheels will stop moving. When the user tilts his hand in the right direction, the gesture is recognized as the right turn, so the right diagonal motors (top right and back right motors) will rotate forwards, and the robot moves in the right direction as shown in Figure 3d. Similarly, when the user tilts his hand in the left direction, the gesture is recognized as the left turn, so only left diagonal motors (top left and back left motors) will rotate forward and the robot moves in the left direction as represented in Figure 3e.

It is worth noting that the values of the tilt of the user's hand determine the right or left turn is a cutting turn. The cutting turn is the one in which without slowing down speed, change its direction. If it is a right turn, the car will start moving in right circle direction, or if it is left turn, the car will move in left circle direction. The hand of a user will never lie within the two gestures (the values for each action is defined differently from another action), i.e., the value of accelerometer will not lie in the threshold values of two directions (left turn and forward, left turn and backward, right turn and forward, right turn and backward).

\subsubsection{Results and Discussions}

In the beginning, the accelerometer (attached with the hand glove) will sense the acceleration forces that are produced with the hand movement at that time, and it will consequently transfer the data to Arduino Nano that is placed on hand glove. After receiving the data, Arduino Nano will convert it into different angle values of ranges $0-450^{\circ}$ and send the data to the RF Receiver of the Arduino Uno that placed on the car through RF Transmitter as clearly exhibited in Figure 4.

When Arduino Uno receives these angles, it will measure these with the predefined set of angle values. Arduino Uno will check if $X_{\text {range }}$ lies between $325^{\circ}-345^{\circ}$ and $Y_{\text {range }}$ lies between $380^{\circ}-410^{\circ}$, it will send a forward signal to motor shield. If Arduino Uno founds $X_{\text {range in }}$ between $315^{\circ}-345^{\circ}$ and Yrange in between $250^{\circ}-275^{\circ}$, it will send a backward signal to motor shield. Similarly, if $X_{\text {range }}$ lies between $385^{\circ}-415^{\circ}$ and $Y_{\text {range }}$ between $315^{\circ}-350^{\circ}$, Arduino will send a Left signal to motor shield. Furthermore, if $X_{\text {range }}$ lies within $255^{\circ}-275^{\circ}$ and $Y_{\text {range }}$ lies within $320^{\circ}-345^{\circ}$, Arduino will send a right signal to motor shield. Thus, after the obtained signal, the motor shield will control the car's movement.

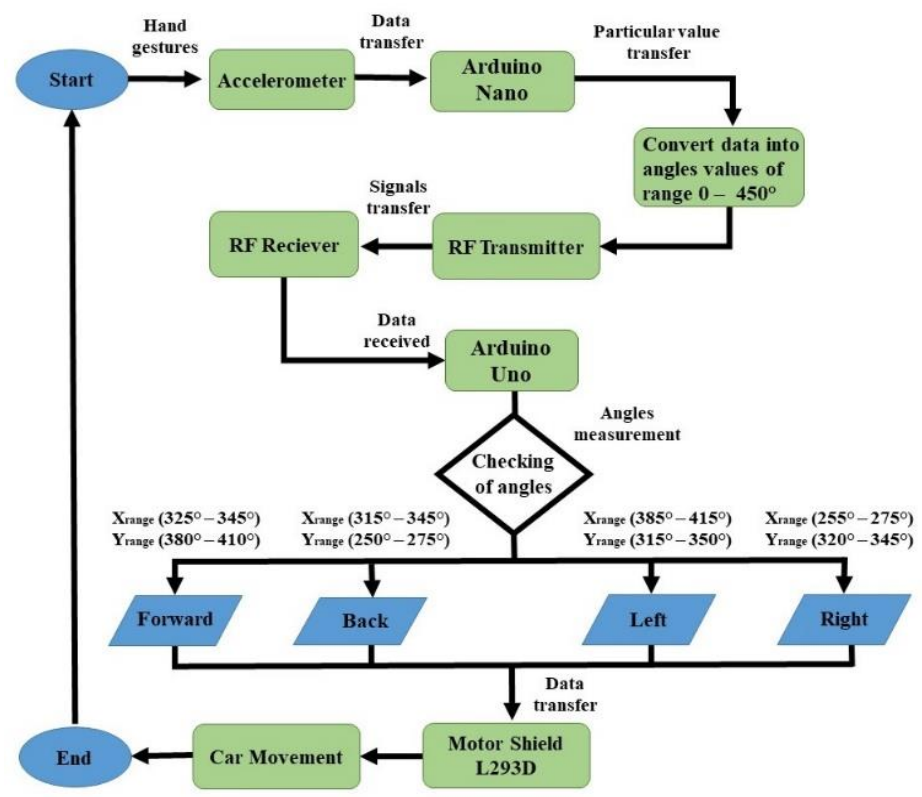

Figure 4. The flow diagram of the hand-gesture based car controlling.

Initially, the robot car will not move and stayed motionless when it does not receive any signal from an accelerometer or when the accelerometer of hand glove is in the straight position. Whenever the user tilts or rotates his hand palm glove to any direction, the angles will be measured of that 
direction by an Arduino and command will send to the motor shield to turn ON corresponding motors and the robot car will start moving in the similar to the hand palm direction.

Figure 5 shows the final presentation of the proposed robot car system that controlled via hand gesture recognition using Arduino. Figure 5a represents the complete hardware implementation of hand glove and robot car. Figure $5 \mathrm{~b}$ shows that the robot car is moving to forward direction (all of the four wheels is moving), Arduino recognized it as a forward command because the hand is bent in the downward position. In Figure 5c, the robot car is moving in backward direction as the hand's position is upwards. In Figure 5d, the robot car is not moving because the hand is in the straight position and Arduino recognized it as brake command. In Figure 5e, the robot car is moving in the right direction (two right diagonal wheels are moving) because the hand is turned in the right position and Arduino considered it as a right command. Similarly, in Figure $5 \mathrm{f}$, the car is moving to the left direction (two left diagonal wheels are moving) because the hand is turned in the left position and Arduino recognized it as a left command.
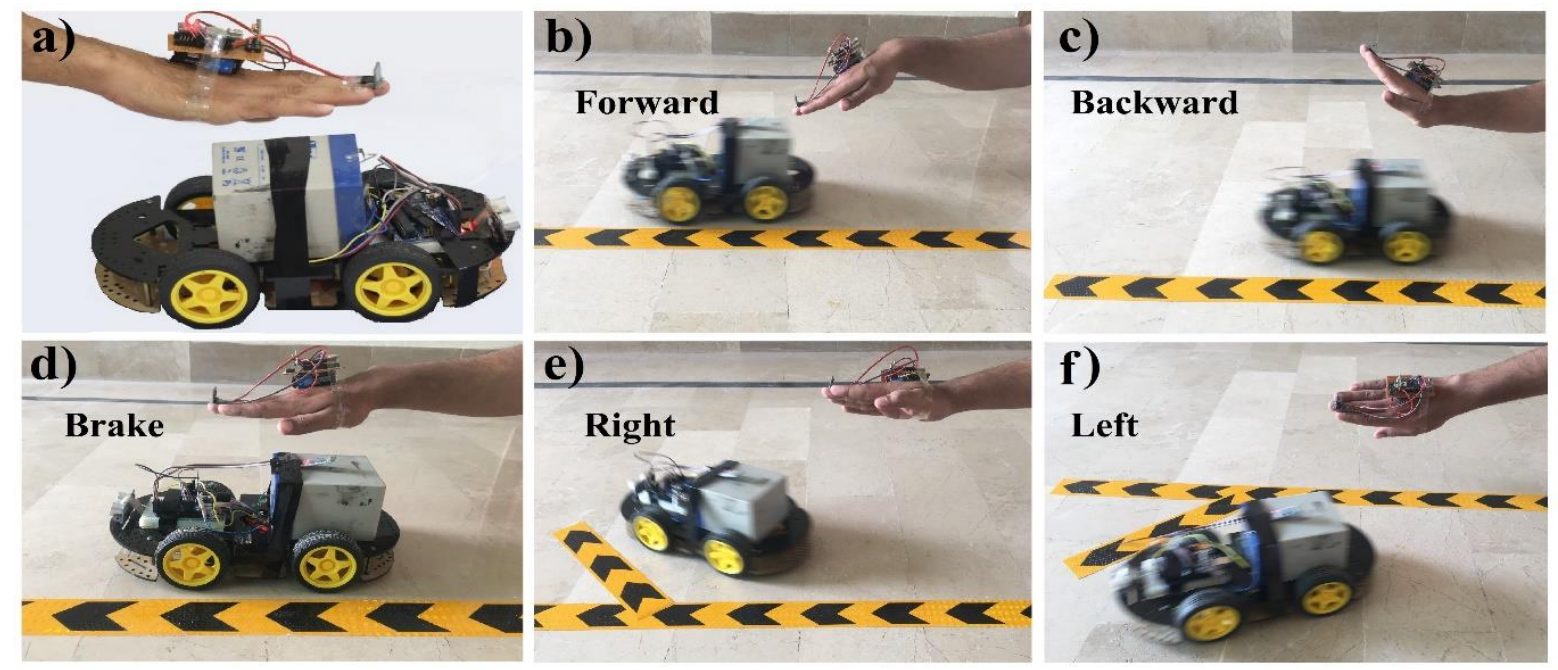

Figure 5. Result diagrams of the automatic robot car controlling system using hand-gestures. (a) Hand gesture and car's hardware are illustrated (b) The hand is tilled in a downward position, so the car is moving forward. (c) The hand is tilted in the upward position, so the car is moving backward. (d) The car stopped when the hand is in a straight position. (e) The car is moving to right direction as the hand-tilted to the right side. (f) The hand is tilted to the left side, so the car is moving to left direction. The complete demonstration video of proposed system is available online [83].

\subsection{Mobile Application System}

According to the motive, the primary idea of this paper is to produce such innovation for the robot car that would be controlled with hand-gesture as well as Android mobile application. As presented in Figure 4, the robot car is controlled via hand-gestures, here, we enhanced our task by controlling the robot car based on Android mobile application. The user can also control the movement of robot car with mobile using Android application (touch button control and voice recognition), and the circuit design of this mode at lab-scale model can be seen in Figure 6 .

In this task, an Arduino UNO, one L298 motor module, one HC-05 Bluetooth module, one 12V battery and four DC motors are used. Hence, TX, RX pin of Bluetooth module is connected to pin number D0, D1, VCC pin to 5V port and ground pin to ground port of the Arduino UNO. Similarly, the $5 \mathrm{~V}$ pin and ground pin of the motor module is connected to $5 \mathrm{~V}$ port and ground port of Arduino $\mathrm{UNO}$. The $12 \mathrm{~V}$ pin of the motor module is connected to positive terminal of a $12 \mathrm{~V}$ battery and negative terminal of the battery is connected to ground port of Arduino, motor module and Bluetooth module. 


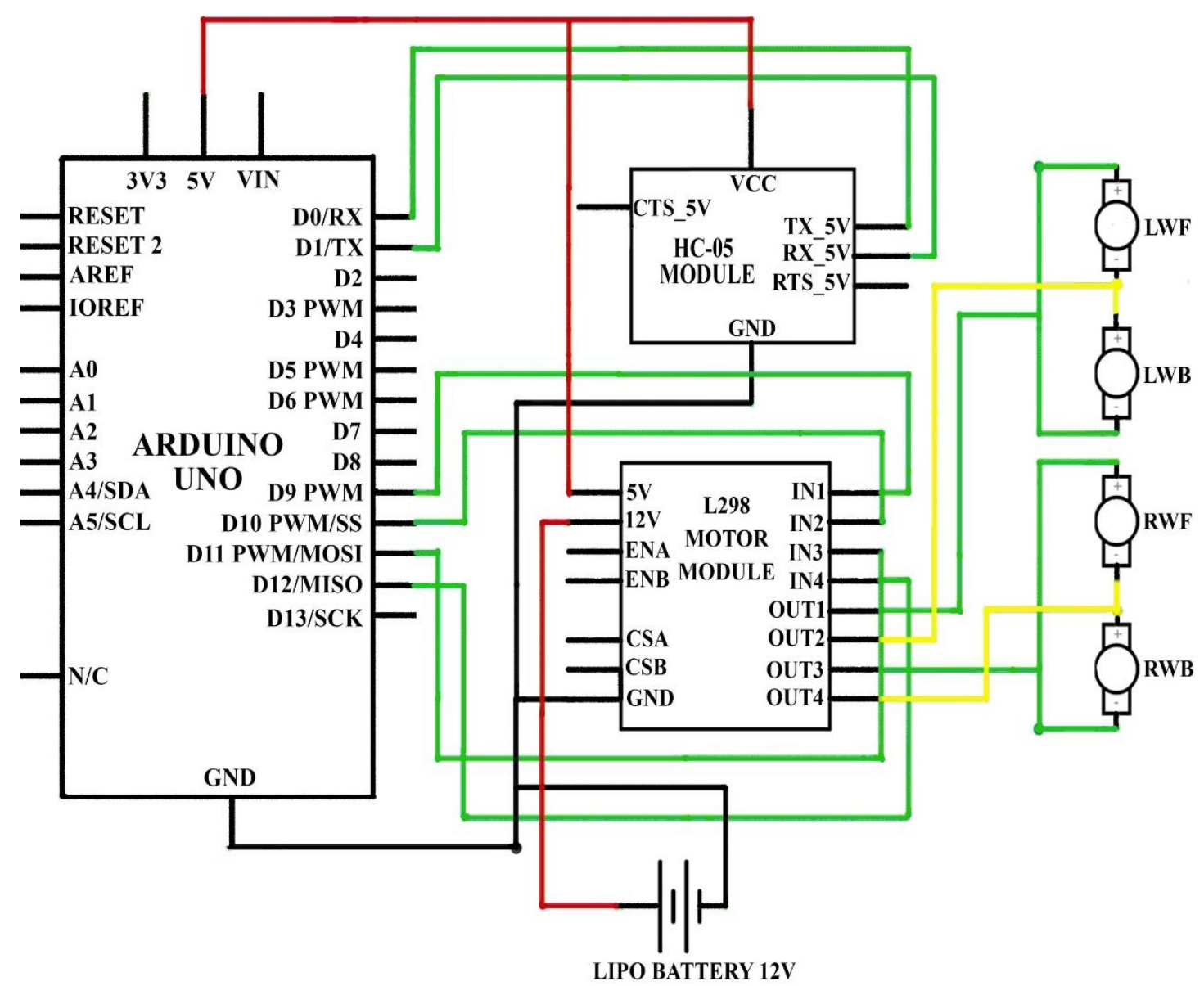

Figure 6. Circuit design of mobile-application to control car.

Moreover, IN1-IN4 pin of the motor module is attached to Arduino PIN D9-D12 respectively. OUT1 pin of motor module is connected to the positive terminal of LWF motor (left wheel forward) and negative terminal of LWB motor (left wheel backward), OUT2 pin to the negative terminal of LWF motor and positive terminal LWB motor, OUT3 pin to positive terminal of the RWF motor (right wheel forward) and negative to the RWB motor (right wheel backward). Likewise, OUT4 pin to the negative terminal of the RWF motor and positive terminal of RWB motor as exhibited in Figure 6 . The complete software code of this case is presented in Figure S2 of the supplementary materials.

\subsubsection{Results and Discussions}

Figure 7 shows the flow diagram to control robot car with an Android-based mobile application. In this scenario, the robot car will move with the built-in Android application with different methods like touch button control (arrow and simple buttons), voice recognition. Firstly, with the touch buttons; after establishing the connection of Android mobile application with Arduino through the Bluetooth module, whenever the user presses any of the touch buttons in the application, the corresponding signal is sent to the Arduino Uno. After receiving the signal, Arduino will check this with predefined instruction for moving forward, backward, left, right, and stop, then send the command to the motor module to move the robot in the corresponding direction.

The second method to control robot car is voice recognition through GUI of android application. When the user selects voice recognition option in the application and speak the specific word (left, right, forward, backward and brake), the application sent this voice command signal to Arduino, and it will start measuring the signal and do operation same as it defined in the previous method to control robot car with touch buttons. 


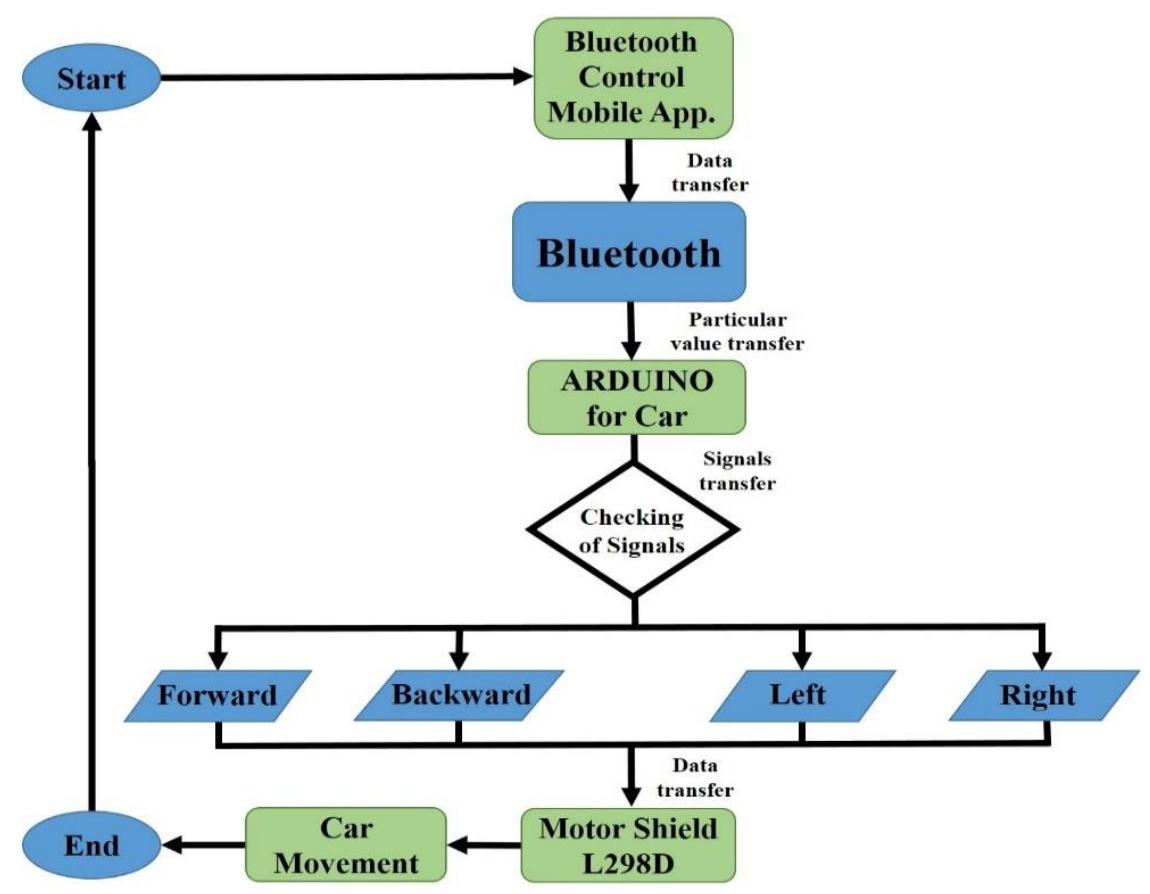

Figure 7. The flow diagram to control robot car with an android mobile application.

Figure 8 shows the result diagrams of the proposed robot car system that controlled via touch buttons of Bluetooth android mobile application using Arduino. In this way, Figure 8a represents the complete working interfaces, where Figure 8a.i is the starting screen of the mobile application; Figure 8a.ii presenting with different controlling interfaces like touch arrow buttons, accelerometer, simple touch buttons and voice recognition etc. Figure 8a.iii showing the four touch arrow buttons; Figure 8a.iv illustrates the six simple buttons (functionality for each button is predefined such as, 1 for forward, 2 for backward, 3 for left, 4 for the right, 5 for stop and six is undefined).

Although, there are five different options to control the car, here, as an example, we used touchbuttons option to demonstrate the working functionality of the proposed system. In Figure 8b, the robot car is moving forward after because the forward touch arrow button is touched in the mobile application. Same as it in Figure $8 \mathrm{c}$, the car is moving backward because the backward touch arrow button is pressed. In Figure $8 \mathrm{~d}$, the car is not moving and stopped at mid-point because none of the touch arrow buttons is pressed. In Figure 8e, the right touch arrow button is touched in the mobile application, and robot car is turned to right direction. Similarly, in Figure 8f, the robot car is moving in the left direction after pressing the left arrow button.

Similarly, Figure 9 shows the result diagrams of the proposed robot car system that is controlled via voice recognition through a Bluetooth android mobile application using Arduino. In this process, Figure 9a represents the voice recognition interface where Figure $9 a . i$ is the primary interface for voice recognition, and Figure 9a.ii shows the interface to input voice after pressing the voice button.

In Figure 9b, the robot car is moving to forward direction after recognition of voice because user inputs the forward move voice command to the mobile application. Similarly, the car is moving in a backward direction after voice recognition because the user inputs the backward move voice command in mobile application as seen in Figure 9c. Meanwhile in Figure 9d, the user input the brake voice command to the application, the robot car is not moving and stopped. On the other hand, in Figure $9 \mathrm{e}$, the robot car is moving in the right direction after recognition of voice because user inputs the right move voice command to the mobile application.

Similarly, the robot car is moving to left direction after recognition of voice because user inputs the left move voice command to the mobile application which can be viewed in Figure 9f. We can also control robot car with built-in accelerometer of mobile application but for testing purposes, we controlled our car with touch buttons and voice recognition. For a quick review, Table 2 summarizes the comparison of proposed systems with old systems. 

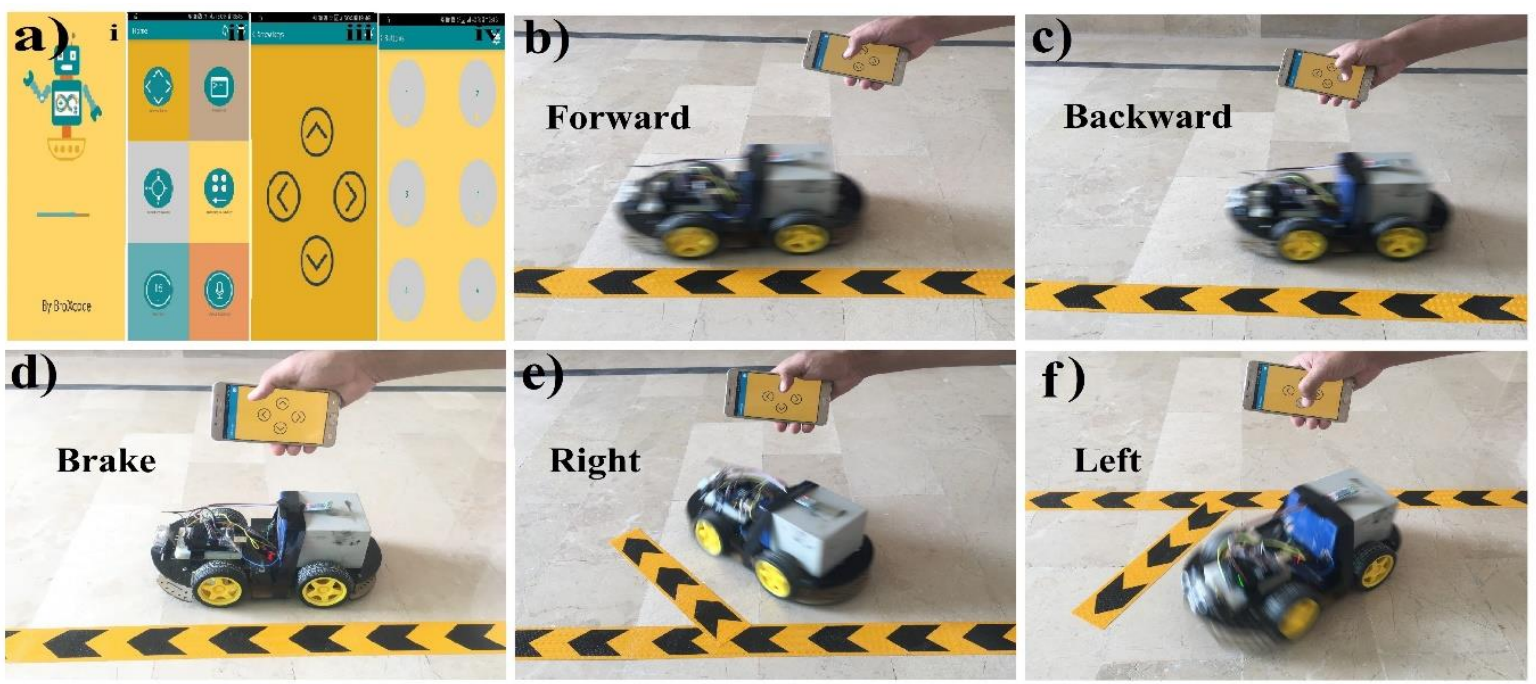

Figure 8. Result diagrams of the automatic robot car controlled with touch buttons of android application. (a) i. Starting interface of the android application. ii. The homepage of an android application showing six different option of car control. iii. Touch arrow buttons interface for controlling car. iv. Simple touch buttons interface for controlling car. (b) The forward arrow button is touched, so the car is moving forward. (c) The backward arrow button is touched, so the car is moving reversely. (d) The car is not moving (stopped) because none of the arrow buttons is touched. (e) The car is moving to right direction as the right arrow button is touched. (f) The left arrow button is touched, so the car is moving to left direction. The complete demonstration video of proposed system is available online [83].
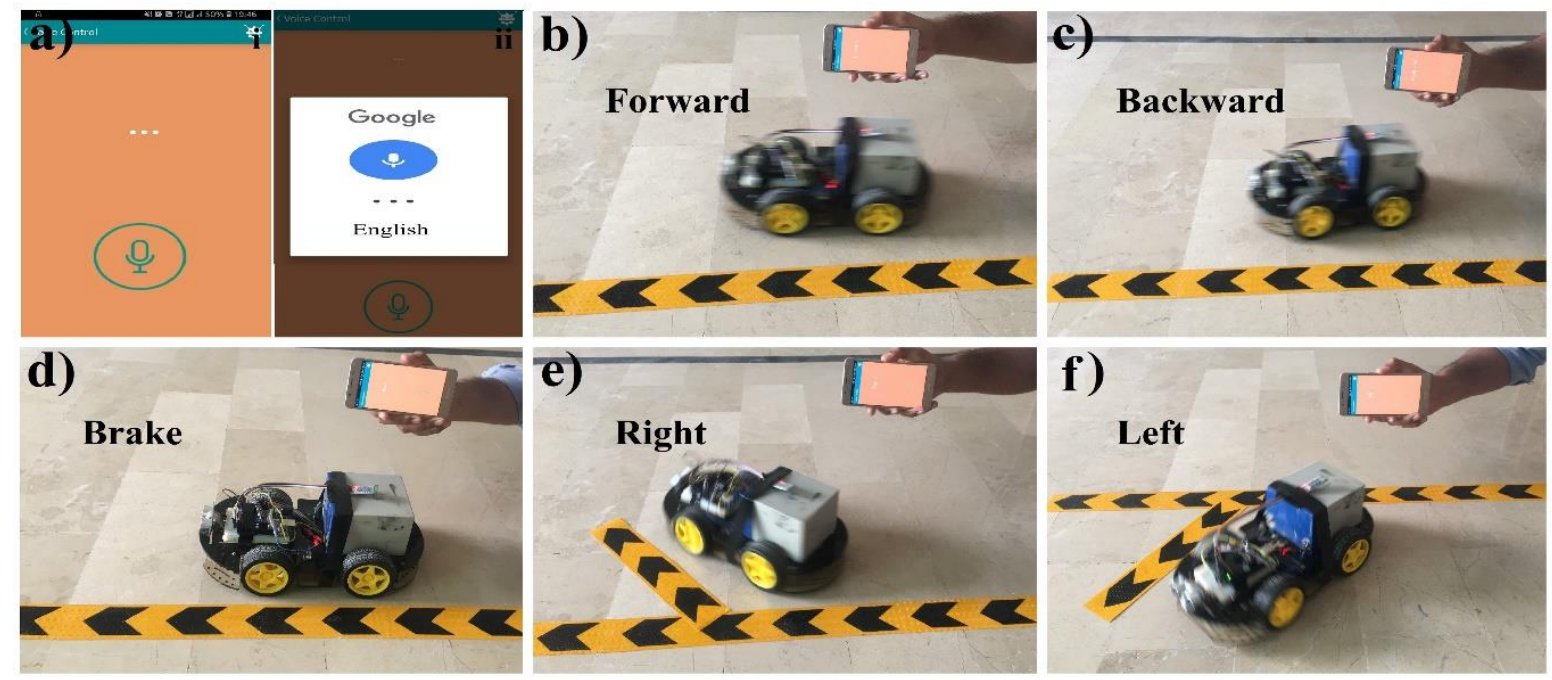

Figure 9. Result diagrams of the automatic robot car controlled with voice recognition of android application. (a) i. Starting interface of voice recognition android application. (a) ii. Voice is recognizing after pressing the voice button of android application. (b) The voice is recognized as a forward command, so the car is moving forward. (c) The voice is identified as a backward command, so the car is moving reversely. (d) The car is not moving (stopped) because the voice is recognized as a brake. (e) The car is moving to right direction as the voice is identified as a right command. (f) The voice is recognized as a left command, so the car is moving to left direction. The complete demonstration video of proposed system is available online [83].

Table 2. Comparison between old systems and the proposed system. 


\begin{tabular}{ccc}
\hline Functionality & Old Systems & Proposed System \\
\hline Based on Arduino & Yes & Yes \\
Operates with gesture and mobile application & Yes & Yes \\
All in one capability ( hand gesture, voice & No & Yes \\
$\begin{array}{c}\text { recognition and touch buttons with mobile } \\
\text { application, Obstacle detection) }\end{array}$ & & \\
\hline
\end{tabular}

We used the Arduino-based control systems with wireless connectivity, in which the robot car could be automatically controlled based on hand gesture recognition and Android application (touch buttons and voice recognition), to avoid any limitations of the specifications, wireless range, etc. In some cases, we required wireless connectivity (WIFI module) to make the system more scalable for easy integration of new devices. Wi-Fi module could be replaced with the Bluetooth module to extend the wireless range for this system further. Moreover, the reported systems are presented as lab-scale prototypes, as aforementioned; therefore, the obstacle detection feature can be implemented for the real-scale facilities to improve safety measurements. Similarly, the proposed systems can be managed for real-scale facilities by applying other technologies like camera [84].

It is worth noting we should consider the cases when the robot car collides with any obstacle in front of it while moving with hand gestures or Bluetooth mobile application. Thus, for improving the safety measurements, we proposed a system to avoid the robot car from a collision with an obstacle by the help of sensor (the car will stop $20 \mathrm{~cm}$ before the obstacle) as illustrated in Figure 10. In Figure $10 \mathrm{a}$, the car is not moving to forward direction whereas the hand is bent to the downward direction and Arduino recognized it as a forward command because the sensor senses an obstacle in front of the car. Similarly, the car is not moving to forward direction although the user pressed the forward arrow button in android mobile application because the sensor senses that there is an obstacle in front of the car, so that is why the car is not moving as illustrated in Figure 10b.

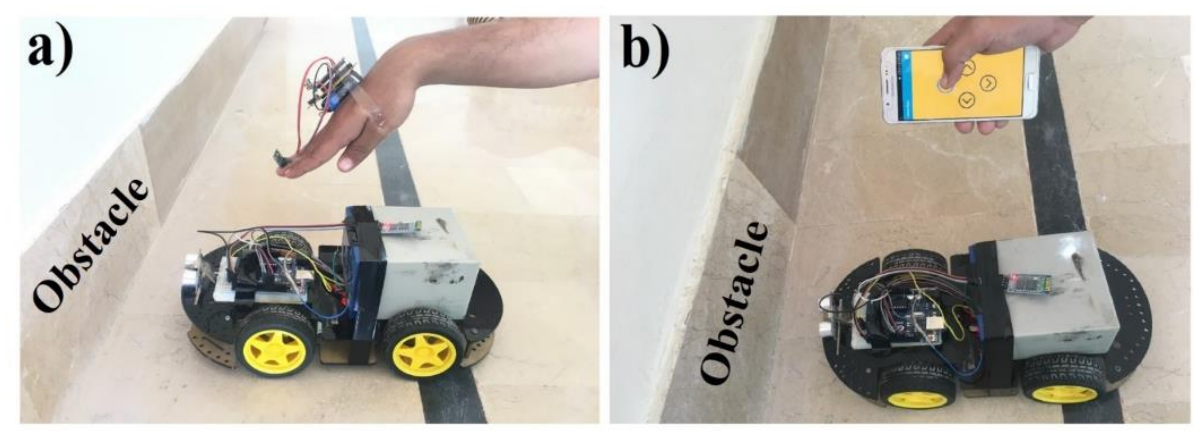

Figure 10. Avoidance of robot car from obstacles using hand-gestures (a) and mobile application (b).

\section{Conclusions}

In this paper, a design scheme for controlling a robot car based on Arduino Uno microcontroller has been demonstrated, which can be programmed to react to events (based on hand gesture recognition and an android mobile application as described above) and to cause corresponding actions. The proposed system presented with principally two operational modes in which the first system is using a mechanism of controlling the robot car based on hand gesture recognition (car moves similarly to the direction and position of hand). This system is further expanded to compose the second mode that controls the robot car based on an android mobile application with GUI touch buttons and voice recognition. Meanwhile, it is presented that the proposed systems have capabilities to control the movement of robot car (forward, backward, left, right and in stopped position). The hardware implementations of the introduced systems were provided at a lab-scale prototype to prove the simplicity, adaptability, dependability, specificity and real low-cost of the system. As a lesson received, we affirmed that the introduced systems could be easily implemented under real requirements at large-scale in the upcoming future, and it can be efficiently implemented in smart cars, personal home robots, articulated robots, parallel robots, humanoid robots, quadrupedal robots, and hybrid robots etc. 
Supplementary Materials: The following are available online at, Figure S1: The circuit design of car controlling system with Arduino using hand gestures recognition. Figure S2: The circuit design of mobile application to control car.

Author Contributions: All of the authors contributed in literature search, figures, study design, data collection, data analysis, data interpretation, and writing etc. Such as, S.U., Z.M., M.A. and H.A.M. designed the devices and carried out the experimental work. S.U., Z.M., S.L., A.M. and H.A.M. analyzed the data and interpreted the results. H.A.M. and Z.M. drafted the manuscript with the input from the others. H.A.M. supervised the project.

Funding: This work is supported by Khwaja Fareed University of Engineering and Information Technology, Rahim Yar Khan, Pakistan. H.A.M. acknowledges the support of the Postdoctoral Science Foundation of China at Southeast University, Nanjing, China, under Postdoctoral number 201557.

Acknowledgments: The authors would like to thank the Editor and the anonymous reviewers for their insightful comments and constructive suggestions that certainly improved the quality of this paper. The authors thank Majid Saleem, Zeeshan Ilyas, Abdullah Zulfiqar from Department of Computer Science, Khwaja Fareed University of Engineering and Information Technology, Rahim Yar Khan 64200, Pakistan and Zaid Mumtaz from Department of Computer Science, Superior University, Lahore, 55150, Pakistan for critical discussions.

Conflicts of Interest: The authors declare no conflict of interest.

\section{References}

1. Qureshi, M.O.; Syed, R.S. The impact of robotics on employment and motivation of employees in the service sector, with special reference to health care. Saf. Health Work. 2014, 5, 198-202.

2. Fernando, Y.A.; Mathath; Murshid, M.A. Improving productivity: a review of robotic applications in food industry. Int. J. Rob. Appl. Technol. 2016, 4, 43-62.

3. Siramshetti, B.K.; Priyanka; Rajitha, C. I won't fall down; Edge detector robot. Int. J. Sci. Eng. Technol. Res. 2017, 6, 113-116.

4. Pakdaman, M.; Sanaatiyan, M.M.; Ghahroudi, M.R. A line follower robot from design to implementation: Technical issues and problems. In Proceedings of the 2nd International Conference on Computer and Automation Engineering (ICCAE), Singapore, 26-28 Feb.2010; pp. 5-9.

5. Singh, R.R.; Das, R.; Pal, K. A novel modus of hand gesture controlled wireless robot. Int. J. Res. Appl. Sci. Eng. Technol. 2017, 5, 641-647.

6. Fang, B.; Sun, F.; Liu, H.; Liu, C. 3D human gesture capturing and recognition by the IMMU-based data glove. Neurocomputing. 2018, 277, 198-207.

7. Shi, Y.; Taib, R.; Lichman, S. Gesturecam: A smart camera for gesture recognition and gesture-controlled web navigation. In Proceedings of the 9th International Conference on Control, Automation, Robotics and Vision, Singapore, 5-8 Dec. 2006; pp. 1-6.

8. Liu, T.; Luo, X.; Liu, J.; Cui, H. Compressive infrared sensing for arm gesture acquisition and recognition. In Proceedings of IEEE International Conference on Information and Automation, Lijiang, China, 8-10 Aug. 2015; pp. 1882-1886.

9. Dahl, T.S.; Gibbons, P.; Jones, O. Identification and production of simple tactile gestures. In Proceedings of IEEE International Conference on Human-Robot Interaction Workshop on Advances in Tactile Sensing and Touch based Human-Robot Interaction, Boston, Massachussetts, Mar. 2012, doi: 10.13140/2.1.3165.344.

10. Cao, S.; Yang, P.; Li, X.; Chen, M.; Zhu, P. iPand: Accurate gesture input with smart acoustic sensing on hand. In Proceedings of the 15th Annual IEEE International Conference on Sensing, Communication, and Networking (SECON), Hong Kong, China, 11-13 June 2018; pp. 1-3, doi: 10.1109/SAHCN.2018.8397157.

11. Jost, C.; De Loor, P.; Nédélec, L.; Bevacqua, E.; Stanković, I. Real-time gesture recognition based on motion quality analysis. In Proceedings of 7 th International Conferences on Intelligent Technologies for Interactive Entertainment (INTETAIN), Turin, Italy, 10-12 June 2015; pp. 47-56.

12. Ju, M.H.; Kang, H.B. Emotional interaction with a robot using facial expressions, face pose and hand gestures. Int. J. Adv. Rob. Syst. 2012, 9, 1-13.

13. Chen, C.-H.; Lee, I.-J.; Lin, L.-Y. Augmented reality-based self-facial modeling to promote the emotional expression and social skills of adolescents with autism spectrum disorders. Res. Dev. Disabil. 2015, 36,396403.

14. Vyas, K.K.; Pareek, A.; Vyas, S. Gesture recognition and control. Int. J. Recent Innovation trends Comput. Commun. 2013, 1,575-581.

15. Vaishnav, P.; Tiwari, S. Accelerometer based hand gesture controlled robot. Int. J. Sci. Res. 2015, 4, $223-226$.

16. Aggarwal, L.; Gaur, V.; Verma, P. Design and implementation of a wireless gesture controlled robotic arm with vision. Int. J. Comput. Appl. 2013, 79, 39-43.

17. Patel, N.K.; Patel, S.B.; Ammar, M.M. Accelerometer based gesture controlled wheel chair with gps, gsm navigation. Int. J. Innovative Emerging Res. Eng. 2015, 2, 110-113. 
18. Goyal, D.; Saini, S.P.S. Accelerometer based hand gesture controlled wheelchair. Int. J. Emerging. Technol. 2013, 4,15-20.

19. Jena, S.P.; Nayak, S.K.; Sahoo, S.K.; Sahoo, S.R.; Dash, S.; Sahoo, S.K. Accelerometer based gesture controlled robot using arduino. Int. J. Eng. Sci. Res. Technol. 2015, 4, 469-475.

20. Budheliya, C.S.; Solanki, R.K.; Acharya, H.D.; Thanki, P.P.; Ravia, J.K. Accelerometer based gesture controlled robot with robotic arm. Int. J. Innovative. Res. Sci. Technol. 2017, 3, 92-97.

21. Mojeebi, T.; Tulo, S.K. Accelerometer gesture controlled robot using arduino. Int. J. Eng. Technol. 2016, 3, 3841.

22. Jayabala, P. Design and implementation of gesture controlled robotic arm for industrial applications. Int. J. Sci. Res. 2018, 3, 202-209.

23. Gupta, C.; Garg, N. Gesture controlled car. Int. J. Electron. Electr. Compute. Syst. 2014, 3, 59-63.

24. P, N.K.; S.R. Hand gesture initiated motor operations for remote robot surveillance system using raspberry pi processor on a zigbee communication link. Int. J. Innovative. Res. Electron. Commun. 2015, 2, 55-62.

25. Jawalekar, P.A. Robot control by using human hand gestures. Int. Res. J. Eng. Technol. 2018, 5, 389-391.

26. Patil, P.V.; Shete, M.B.; Padalkar, T.M. Wireless hand gesture robot using accelerometer. Int. Res. J. Eng. Technol. 2016, 3, 353-356.

27. Verma, S. Hand gestures remote controlled robotic arm. Adv. Electron. Electr. Eng. 2013, 3, 601-606.

28. Suriya, T.S.U.; Khan, S.; Selvaganapathy, S.; Solaimani.; Prasannakumar, V. Gesture controlled prosthetic arm. Int. J. Adv. Res. Dev. 2017, 2, 56-59.

29. Jhaveri, R.B.; Mehta H.M.; Gala, P.T. Gesture controlled robot. Int. J. Electron. Electr. Compute. Syst. 2015, 4, 15-19.

30. Rao, S.; Rajasekhar, C. Password based hand gesture controlled robot. Int. J. Eng. Res. Appl. 2016, 6, 63-69.

31. Setia, A.; Mittal, S.; Nigam, P.;Singh, S.; Ganwar, S. Hand gesture recognition based robot using accelerometer sensor. Int. J. Adv. Res. Electr. Electron. Instrum. Eng. 2015, 4, 4470-4476.

32. N, S. Design and implementation of accelerometer based robot motion and speed control with obstacle detection. Int. J. Sci. Eng. Technol. 2013, 2, 749-755.

33. Mishra, A.; Malhotra, S.; Singh, H.P.; Ruchira. Design of hand glove for wireless gesture control of robot. Int. J. Pure. Appl. Math. 2017, 114, 69-79.

34. Verma, S. Android app controlled bluetooth robot. Int. J. Comput. Appl. 2016, 152, 35-40.

35. Maity, A.; Paul, A.; Goswami, P.; Bhattacharya, A. Android application based bluetooth controlled robotic car. Int. J. Intell. Information.Syst. 2017, 6, 62-66.

36. Pahuja, R.; Kumar, N. Android mobile phone controlled bluetooth robot using 8051 microcontroller. Int. J. Sci. Eng. Res. 2014, 2, 14-17.

37. Kumar, R.;p, U.; Kubade, p.R.; Kulkarni, H.B. Android phone controlled bluetooth robot. Int. Res. J. Eng. Technol. 2016, 3, 104-114.

38. Roy, S.; Wangchuk, T.P.; Bhatt, R. Arduino based bluetooth controlled robot. Int. J. Eng. Trends. Technol. 2016, 32, 216-219.

39. Kanere, S.; Shinde, R.; Tornekar, V.; Zagade, D.; Sonar, V.S. Bluetooth controlled car system. Int. J. Adv. Res. Comput. Commun. Eng. 2017, 6, 44-46.

40. Gandotra, S.; Sharma,B.; Mahajan, S.; Motup, T.; Choudhary, T.; Thakur, P. Bluetooth controlled rc car using arduino. Int. J. Interdiscip. Res. 2016, 2, 144-147.

41. Rai, N.; Rasaily, D.; Wangchuk, T.R.; Gurung, M.; Khawas, R.K. Bluetooth remote controlled car using arduino. Int. J. Eng. Trends. Technol. 2016, 33, 381-384.

42. Kannan, K.; Selvakumar, J. Arduino based voice controlled robot. Int. Res. J. Eng. Technol. 2015, 2, 49-55.

43. Rashid, H.; Ahmed, I.U.; Osman, S.B.; Newaz, B.; Rasheduzzaman, M.; Reza, S.M.T. Design and implementation of a voice controlled robot with human interaction ability. International Conference on Computer, Communication, Chemical, Materials and Electronic Engineering, 2017, 65, 148-151.

44. Memon, Y.A.; Motan, I.; Akbar, M.A.; Hameed, S.; Hasan, M.U. Speech recognition system for a voice controlled robot with real time obstacle detection and avoidance. Int. J. Electr. Electron. Data. Commun. 2016, 4, 33-37.

45. Zope, S.; Muluk, P.; Mohite, R.; Lanke, A.; Bamankar, M. Voice control robot using android application. Int. J. Interdiscip. Res. 2017, 3, 1723-1726.

46. Harshad, P.; Amol, J.; Dnyaneshwar, K.; Bhosale, A.C. Voice control robot using arduino pick and place object. Int. J. Innovations. Eng. Res. Technol. 2017, 4, 27-30.

47. Kumar, A.; Chauhan, R. Voice controlled robot. Int. J. Innovative. Res. Technol. 2014, 1, 338-344.

48. Saravanan, D.; Parthiban, R.; Archanaa, G.I. Voice controlled robotic car using arduino for smart agriculture. Int. J. Pure. Math. 2018, 118, 2097-2105.

49. Chikhale, V.; Gharat, R.; Gogate, S.; Amireddy, R. Voice controlled robotic system using arduino microcontroller. Int. J. New. Technol. Res. 2017, 3, 92-94.

50. Louis, L. Working principle of arduino and using it as a tool for study and research. Int. J. Control Autom. Commun. Syst. 2016, 1, 21-29. 
51. Punetha, D.; Kumar, N.; Mehta, V. Development and applications of line following robot based health care management system. Int. J. Adv. Res. Compute. Technol. 2013, 2, 2446-2450.

52. O, E.O.; A, A.Y.; A, A.L.; D, O.O. Line follower robot using a sophisticated sensor approach. Int. J. Eng. Res. Technol. 2013, 2, 1980-1982.

53. Gumus, O.; Topaloglu, M.; Ozcelik, D. The use of computer controlled line follower robots in public transport. 12th International Conference on Application of Fuzzy Systems and Soft Computing, 2016, 102, 202-208.

54. Mi, J.; Takahashi, Y. An design of hf-band rfid system with multiple readers and passive tags for indoor mobile robot self-localization. Sens, 2016, 16, 1-20, 1200.

55. Chavan, A.S.; Pansare, D.D.; Kadam, S.P.; Mayekar, N.K.; Jha, K.V.; Bhagwat, P.R. Design of accident prevention system using QRD 1114 and CNY 70 Sensors. Int. J. Emerging. Technol. Adv. Eng. 2013, 3, 525-530.

56. Mohamed, A.; Yang, C.; Cangelosi, A. Stereo vision based object tracking control for a movable robot head. International Federation of Automatic Control, 2016, 49, 155-162.

57. Pati, C.S.; Kala, R. Vision-based robot following using pid control. Technol., 2017, 5, 1-17, 3390.

58. Nadiger, N.; K, M.; Naik, S. humanoids implementation using sixth sense. Int. J. Inf. Syst. Compute. Sci. 2012, 1, 31-34.

59. Purohit, R.; Vyas, S.; Mathur, P. Ai and its application: sixth sense technology. Int. J. Emerging. Trends. Technol. Compute. Sci. 2013, 2, 184-186.

60. Daroga, R.; Pandey, N. Sixth Sense Technology \& Its Applications. Int. J. Sci. Res. Publ. 2015, 5, 1-4.

61. V, D.; D, j.; R, v. Zigbee based wireless intelligent security robot for defence camp. Int. J. Adv. Eng. Global. Technol. 2014, 2, 576-583.

62. Patel, A.; Chaudhari, K.; Patel, D. Touch screen controlled multipurpose spy robot using zigbee. Int. J. Adv. Res. Compute. Eng. Technol. 2014, 3, 1058-1062.

63. M, P. Unmanned multi-functional robot using zigbee adopter network for defense application. Int. J. Adv. Res. Compute. Eng. Technol. 2013, 2, 47-55.

64. Song, B.; Lu, X.; Bai, X. Zigbee based wireless sensor and actuator network for service robot intelligent space. Wireless Sens. Network. 2012, 4, 235-242.

65. Iswarya, P.; Ramarao, D.; Kumar, B.D.; Kumar, K.D.; Manikyalarao, T. Obstacle aviodance robot using arduino. Int. J. Adv. Res. Sci. Eng. 2017, 6, 634-638.

66. Bhagat, K.; Deshmukh, S.; Dhonde, S.; Ghag, S. Obstacle Avoidance Robot. Int. J. Sci. Eng. Technol. Res. 2016, 5, 439-442.

67. Ankit, V.; Jigar, P.; Savan, V. Obstacle avoidance robotic vehicle using ultrasonic sensor, android and bluetooth for obstacle detection. Int. Res. J. Eng. Technol. 2016, 3, 339-348.

68. Dumbre, K.; Ganeshkar, S.; Dhekne, A. Robotic vehicle control using internet via webpage and keyboard. Int. J. Comput. Appl. 2015, 114, 15-19.

69. Kadir, W.M.H.W.; Samin, R.E.; Ibrahim, B.S.K. Internet controlled robotic arm. Int. Symp. Rob. Intell. Sens. 2012, 41, 1065-1071.

70. Patil, A.D.; Kadiri, H.I.; Joshi, A.S.; Wani, A.B. IOT based remote access human control robot using mems sensor. Int. J. Comput. Sci. Mobile. Comput. 2016, 5, 816-826.

71. Kalaiarasi, D.; S, P.; S, P.; L, P.R. Iot based motion control system of a robotic car. Int. Res. J. Eng. Technol. 2018, 5, 3073-3076.

72. M, N.K.; P, P.A.; M, V.k.; R, M.; N, G.M.; B, K. Iot based smart car parking using line following robot. Int. J. Electr. Electron. Data. Commun. 2017, 5, 46-48.

73. Nayyar, A.; Puri, V.; Nguyen, N.G.; L, D.N. Smart surveillance robot for real-time monitoring and control system in environment and industrial applications. Inf. Syst. Des. Intell. Appl., Adv. Intell. Syst. Comput. 2018, 229-243, 10.1007/978-981-10-7512-4_23.

74. J, A.; Abraham, A.M.; George, L.M.; Saji, V.A.; R, A.A. Smart robotic assistant using iot. Int. Res. J. Eng. Technol. 2017, 4, 857-859.

75. Google play. Arduino Bluetooth Control. Available online: https://play.google.com/store/apps/details?id=com.broxcode.arduinobluetoothfree\&hl=en (accessed on 31 December 2018).

76. Arduino Uno Rev3. Available online: https://store.arduino.cc/usa/arduino-uno-rev3 (accessed on 31 December 2018).

77. Compenets101. Arduino Nano. Available online: https://components101.com/microcontrollers/arduinonano (accessed on 31 December 2018).

78. SunRom. Gyro + Accelerometer Sensor, 3 Axis based on MPU-6050. Available online: https://www.sunrom.com/p/gyro-accelerometer-sensor-3-axis-based-on-mpu-6050 (accessed on 31 December 2018).

79. ElProCus. RF Module - Transmitter \& Receiver. Available online: https://www.elprocus.com/rf-moduletransmitter-receiver/ (accessed on 31 December 2018). 
80. SunFounder. L293D Motor Driver Shield. Available online: http://wiki.sunfounder.cc/index.php?title=L293D_Motor_Driver_Shield (accessed on 31 December 2018).

81. Itead. Serial Port Bluetooth Module HC-05. Available online: https://www.itead.cc/wiki/Serial_Port_Bluetooth_Module_(Master/Slave)__HC-05 (accessed on 31 December 2018).

82. How to Mechatronics. L298 Motor Module. Available online: https://howtomechatronics.com/tutorials/ arduino/arduino-dc-motor-control-tutorial-1298n-pwm-h-bridge/ (accessed on 31 December 2018).

83. Youtube. An Automated Robot Car Control System with Hand Gestures and Mobile Application Using Arduino. Available online: https://www.youtube.com/watch? $=3$ Nqz9jewack\&feature=youtu.be (accessed on 31 December 2018).

84. Insteon. Insteon WiFi Cameras. Available online: https://www.insteon.com/wifi-cameras/ (accessed on 31 December 2018). 